\title{
Quantifying photon recycling in solar cells and light emitting diodes: absorption and emission are always key
}

Alan R. Bowman ${ }^{1}$, Miguel Anaya ${ }^{1}$, Neil C. Greenham ${ }^{1} \&$ Samuel D. Stranks ${ }^{1,2 *}$

1. Cavendish Laboratory, Department of Physics, University of Cambridge, J.J. Thomson Avenue, Cambridge, CB3 OHE, UK

2. Department of Chemical Engineering \& Biotechnology, University of Cambridge, Philippa Fawcett Drive, Cambridge, CB3 0AS, UK

*Corresponding author: $\underline{\text { sds65@cam.ac.uk }}$

\begin{abstract}
Photon recycling has received increased attention in recent years following its observation in halide perovskites. It has been shown to lower the effective bimolecular recombination rate and thus increase excitation densities within a material. Here we introduce a general framework to quantify photon recycling which can be applied to any material. We apply our model to idealized solar cells and light-emitting diodes based on halide perovskites. By varying controllable parameters which affect photon recycling, namely thickness, charge trapping rate, non-ideal transmission at interfaces, and absorptance, we quantify the effect of each on photon recycling. In both device types, we demonstrate that maximizing absorption and emission processes remains paramount for optimizing devices, even if this is at the expense of photon recycling. Our results provide new insight into quantifying photon recycling in optoelectronic devices and demonstrate that photon recycling cannot always be seen as a beneficial process.
\end{abstract}

\section{Main}

Photon recycling has been reported in luminescent semiconductors including GaAs, InP and, more recently, halide perovskites such as methylammonium lead iodide $\left(\mathrm{MAPbI}_{3}\right)$ [1-4]. It is a direct consequence of the reciprocity between absorption and emission - if a material can emit a photon at a wavelength it can also absorb a photon at that wavelength $[5,6]$. The role of photon recycling in decreasing radiative recombination rates in perovskites has been discussed [7,8]. Recently, Brenes et al. explored the effect of photon recycling on the maximum power point voltage in idealized perovskite solar cells with variable charge trapping rates [9] and demonstrated an increase in maximum power point voltage of $77 \mathrm{mV}$ can be attributed to photon recycling. Similarly, Cho et al. recently showed photon recycling to be of importance in perovskite light-emitting diodes [4]. 
Here, we construct a generalized framework to quantify photon recycling. We make no assumptions about the nature of the excitation, the emission or the competing loss processes. We calculate the number of photon recycling events per initial excitation, $N$, (from external photon absorption or charge injection) under different device-relevant conditions, allowing us to draw conclusions as to when the phenomenon is most beneficial. We apply our model to idealized $\mathrm{MAPbI}_{3}$ solar cells and $\mathrm{CsPbBr}_{3}$ LEDs at operating voltages and quantify the effect of different controllable parameters. For solar cells, we go beyond previous reports [9] by considering changes in both current and voltage, and their relation to controllable parameters. In both device types, increasing thickness, improving reflection at the rear interface and decreasing charge trapping all enhance device performance and $N$. However, we demonstrate that enhancements due to photon recycling do not compensate for reduced light absorption or emission when considering transmission at the front interface and surface roughness. We conclude that to maximize solar cell or LED performance, one must maximize absorptance or emittance, even if this is at the expense of photon recycling.

We begin by introducing the probability an emitted photon of energy $E$ escapes a material, $\eta_{\text {esc }}(E)$, which is given by

$$
\eta_{e s c}(E)=\frac{a(E)}{4 n(E)^{2} \alpha(E) t}
$$

Here $n(E)$ is the (real) refractive index, $\alpha(E)$ the absorption coefficient and $t$ thickness. To derive this we assume negligible Stokes shift (corresponding to maximized photon recycling), so the absorptance, $a(E)$, is equal to the emissivity [5] (see derivation in Supplementary Note 2). Subsequent conclusions do not rely on this assumption. Equation 1 demonstrates maximizing absorptance also maximizes $\eta_{e s c}(E)$ and therefore can reduce $N$.

As the number of photons emitted at energy $E$ (per unit volume, per unit energy, per unit time) is $4 \pi n(E)^{2} \alpha(E) \Phi_{b b}(E) e^{\frac{\mu}{k_{B} T}}[10]$, the energy-averaged escape probability is expressed by

$$
\bar{\eta}_{e s c}=\frac{\int(\text { No.of photons emitted at } E) \times \eta_{e s c}(E) d E}{\int(\text { No.of photons emitted at } E) d E}=\frac{\int \pi a(E) \Phi_{b b}(E) d E}{t \int 4 \pi n(E)^{2} \alpha(E) \Phi_{b b}(E) d E}
$$


in agreement with derivations from thermodynamic considerations [6]. Here $\Phi_{b b}(E)$ is the photon flux emitted by the surface of a black body into air (per unit area, per unit solid angle, per unit energy), $\mu$ the quasi Fermi-level splitting and $k_{B} T$ the thermal energy.

All excitations undergo either radiative or non-radiative decay, with (internal) rates $R_{r}$ and $R_{n r}$. We define internal photoluminescence quantum efficiency, $P L Q E_{\text {int }}$ (or, for electrical injection, internal electroluminescence quantum efficiency), as the ratio of radiative recombination to total recombination,

$$
P L Q E_{\text {int }}=\frac{R_{r}}{R_{r}+R_{n r}}
$$

The probability of a photon re-absorption event occurring is

$$
p=P L Q E_{\text {int }}\left(1-\bar{\eta}_{\text {esc }}\right)
$$

A directly measurable quantity is the external PLQE (PLQE ext $)$, the ratio of escaping photons to absorbed incident external excitation [11]. Under steady state illumination, the decay of excitations is balanced by local generation: $G=R_{r}+R_{n r}$. However, generation is from both incident and recycled photons, thus $G=G_{\text {ext }}+\left(1-\bar{\eta}_{e s c}\right) R_{r}$. Assuming uniform illumination and uniform excitation density,

$$
P L Q E_{\text {ext }}=\frac{\text { No.of escaping photons }}{G_{\text {ext }}}=\frac{\bar{\eta}_{e s c} R_{r}}{\bar{\eta}_{e s c} R_{r}+R_{n r}}=\frac{\bar{\eta}_{e s c} P L Q E_{\text {int }}}{1-\left(1-\bar{\eta}_{e s c}\right) P L Q E_{\text {int }}} .
$$

For a process with probabilities $p_{i}$ and outcomes $X_{i}$, the weighted average is $\sum_{i} p_{i} X_{i}$. Therefore, the average number of photon recycling events per initial excitation is

$$
N=\sum_{i=0}^{\infty} i p^{i}(1-p)=\frac{p}{(1-p)}=P L Q E_{\text {ext }}\left(\frac{1}{\bar{\eta}_{\text {esc }}}-1\right)
$$

We plot $N$ as a function of $P L Q E_{\text {ext }}$ and $\bar{\eta}_{\text {esc }}$ in Figure 1, where the dashed line indicates $N=1$. This plot is applicable to any material and allows one to assess whether photon recycling is a 
dominant process (noting $P L Q E_{\text {ext }}$ and $\bar{\eta}_{\text {esc }}$ should be measured or calculated for the specific material). For $N=1$ we require $\bar{\eta}_{e s c}<50 \%$ and high $P L Q E_{e x t}$, while for materials with large Stokes shift, such as many organic semiconductors, $\bar{\eta}_{e s c} \rightarrow 100 \%$, thus there is very little photon recycling (note equations 1 and 2 will need modification in case of large Stokes shift).

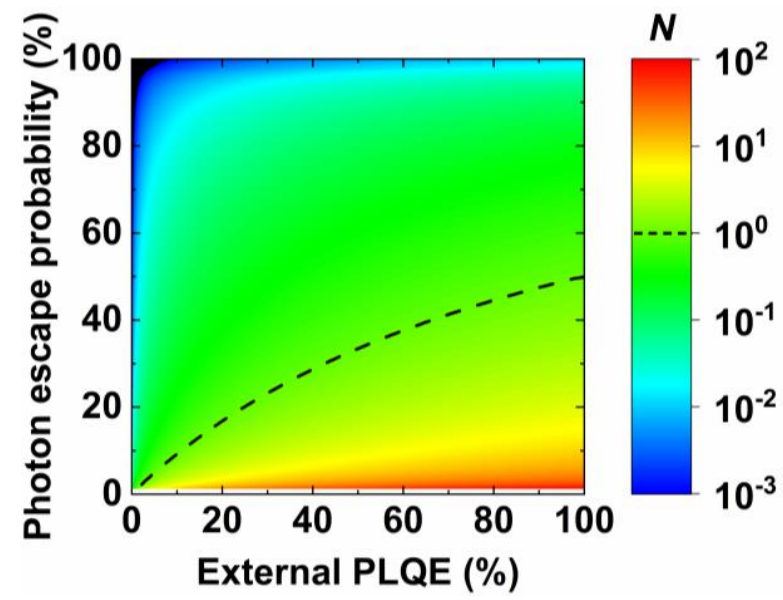

Figure 1. The number of photon recycling events per initial excitation $(N)$ as a function of the external PLQE (PLQE ext) and the escape probability $\left(\bar{\eta}_{\text {esc }}\right)$. Dashed line marks one photon recycling event per initial excitation.

We now quantify photon recycling in solar cells and LEDs. For solar cells, we use MAPbI3 from the recently emerged class of halide perovskites. $\mathrm{MAPbI}_{3}$ has small Stokes shift [12], allowing for computation of $\bar{\eta}_{e s c}$ from equation 2. Following the approach of Pazos-Outón et al. [13] we consider $\mathrm{MAPbI}_{3}$ an intrinsic semiconductor under $\mathrm{AM} 1.5$ with recombination due to charge trapping, radiative and Auger processes (see Supplementary Note 3). Unless otherwise stated, our solar cell interacts with radiation from a $2 \pi$ hemisphere, has a perfect back reflector and no parasitic absorption. We explore four controllable parameters which affect photon recycling: thickness; charge trapping; front and back transmission coefficients; and absorptance (depending on light trapping). While these parameters may be inter-related in any actual solar cell, decoupling these phenomena allows us to quantify their effects on photon recycling. For thickness, trapping rate and transmission coefficients we use a Beer-Lambert absorptance model (termed Direct), though we show similar results from other models in Figure S1-11.

We plot the number of photon recycling events at the maximum power point, $N_{m p p}$, as a function of thickness with no charge trapping in Figure 2a. We carried out simulations up to film thicknesses of 1000-nm because charge diffusion lengths are likely to become limiting at larger thicknesses [12]. Even in this idealized case of no trapping, $N_{m p p}<1$, primarily because the majority of charges are extracted to the external circuit before they can recombine; at opencircuit there can be over 10 photon recycling events (Figure 2a, inset). As thickness increases 
efficiency and $N_{m p p}$ increase (Figures S3 and S4). Efficiency rises due to an increase in shortcircuit current, (Figure S5a) while $N_{m p p}$ increases because $\bar{\eta}_{e s c}$ decreases significantly (Figure S6), while $P L Q E_{\text {ext }}$ is relatively flat (Figure $S 7$ ).
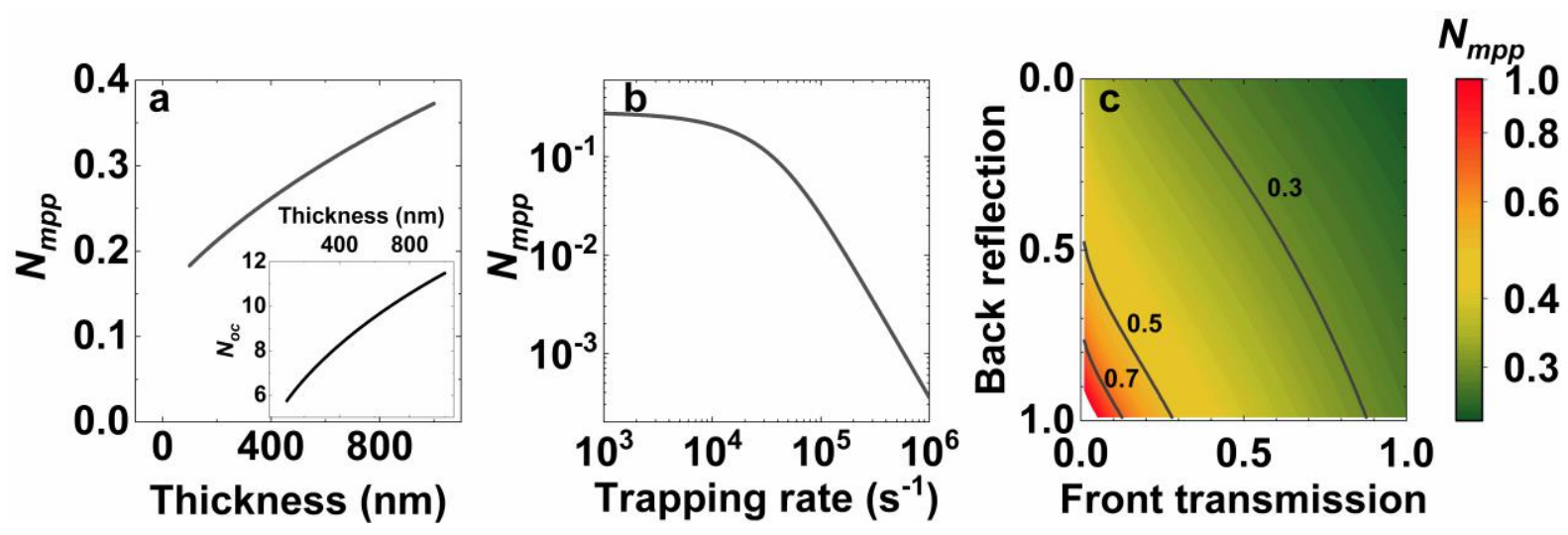

Figure 2. Number of photon recycling events per absorbed incident photon at maximum power point $\left(N_{\text {mpp }}\right)$ as a function of: a) thickness (with no charge trapping); and b) charge trapping for a $\mathrm{MAPbI}_{3}$ solar cell. Inset in a) shows the number of photon recycling events at open-circuit voltage $\left(N_{o c}\right)$ for the same system as in a). c) $N_{m p p}$ with non-ideal front transmission and back reflection (no charge trapping). Calculations b) and c) are performed on a 500-nm thick film.

We show $N_{m p p}$ for a 500-nm thick absorber layer in Figure 2b. $N_{m p p}$ falls rapidly from values in Figure 2a unless charge trapping rates are $<10^{4} \mathrm{~s}^{-1}$ (current state of the art is $1 \times 10^{5} \mathrm{~s}^{-1}$, where $N_{m p p} \sim 0.025$ [14]). As charge trapping is decreased, open-circuit voltage increases (Figure $\mathrm{S} 5 \mathrm{~b}$ ), corresponding to gains in efficiency and $N_{m p p}$ due to reduced non-radiative recombination (Figure S9). While it is possible for trapping rate to be a function of applied bias [15], here we assume it constant, as we are primarily interested in maximized photon recycling (where charge trapping has minimal effect). Our results show that, for fixed absorptance, reducing charge trapping increases $N_{m p p}$ and efficiency.

We next consider non-ideal front transmission and back reflection, both of which can also correspond to parasitic absorption. $N_{m p p}$ varies from 0.2 to 1 for front transmission coefficient larger than $5 \%$ (Figure 2c) but reduces at higher efficiencies $\left(N_{m p p}<0.3\right.$ when efficiency $>$ $26.5 \%$, Figure S10). $N_{m p p}$ is more strongly affected by front transmission than rear reflection as reducing front transmission lowers the excitation density, reducing the effects of nonradiative Auger recombination. Importantly, $N_{m p p}$ reduces as front transmission (and consequently efficiency) are improved.

We consider the effect of light management (necessary for any optimized solar cell) on photon recycling by considering three absorptance models (Supplementary Table 1 and Figure S12). 
These represent a flat surface, a roughened surface, and an idealized surface whereby the film absorbs the maximum light possible for a given thickness, termed Direct, Randomized and Maximal, respectively. We consider a 500-nm thick $\mathrm{MAPbI}_{3}$ solar cell for our three different absorptances and vary the charge trapping rate. In Figure 3a we plot $N_{m p p}$ versus efficiency, both as a function of the first-order trapping rate, for all absorptances. Randomized and Maximal absorptances give higher efficiencies than Direct for the same trapping rates as they lead to more light absorption. Importantly, models that yield higher efficiency also give lower $N_{m p p}$, due to increased escape probabilities. For example, with a loss rate of $10^{5} \mathrm{~s}^{-1}$, Direct gives an efficiency of $27.8 \%$ and $N_{m p p}=0.025$, while Maximal yields an efficiency of $29.8 \%$ and $N_{m p p}=0.015$. Randomized and Maximal models yield a decrease in open circuit voltage (due to more re-emitted light), which is more than compensated for by an increase in the short circuit current. This is demonstrated in the current-voltage curves shown in Figure $3 b$ (see further discussion in Supplementary Note 4). We note that the interplay between charge trapping and absorptance (treated independently here) should be considered when experimentally optimizing solar cells.
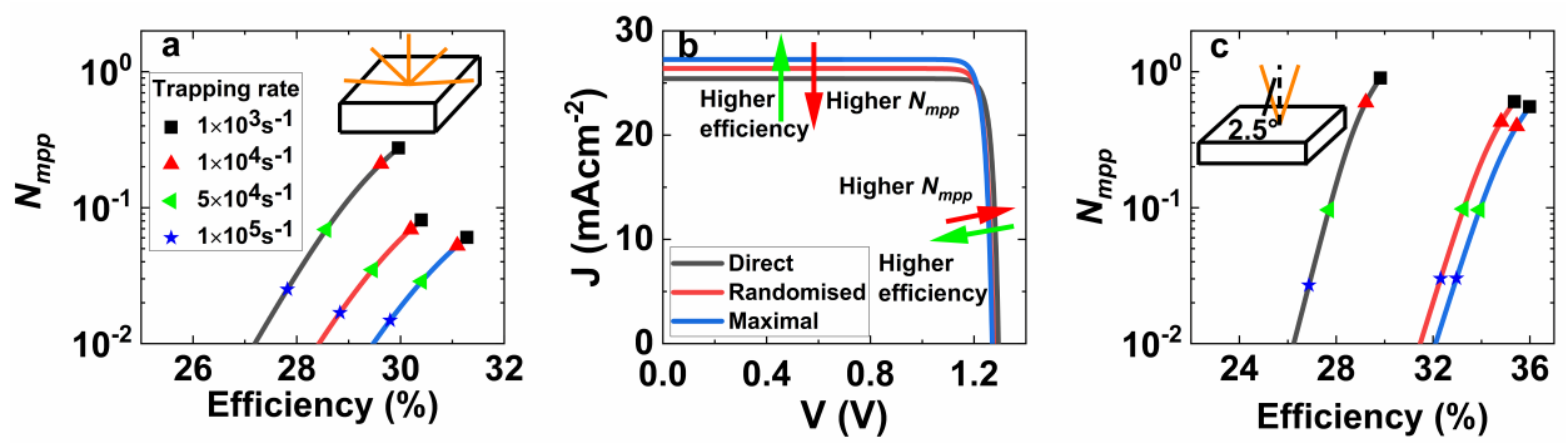

Figure 3. a) Relationship between the number of photon recycling events at maximum power point, $N_{m p p}$, and efficiency (for interaction with a full $2 \pi$ hemisphere, see schematic) for $M A P b I_{3}$ solar cells in which the charge trapping rate (selected values defined in legend) is varied for different absorptance models (legend in b) in a 500-nm thick film. b) Current-voltage characteristics for the three absorptance models considered, with no charge trapping. c) Results equivalent to a) for a $\mathrm{MAPbI}_{3}$ solar cell which only interacts with $2.5^{\circ}$ of direct and circumsolar radiation (see schematic).

A second approach to controlling absorptance is reducing the solid angle of interaction with the surroundings, reducing re-emitted light [16-18]. Therefore, we consider a solar cell which only interacts with direct and circumsolar light and present corresponding results for the three absorptance models in Figure $3 \mathrm{c}$ using reciprocity to calculate absorptance and emittance. We 
note circumsolar and direct AM1.5 contains less blue light, slightly reducing short circuit currents (Figure S13). Therefore, for Direct absorptance we see a slight decrease in the maximum efficiency (from $30.0 \%$ to $29.9 \%$ ), and $N_{m p p}=0.94$ with no charge trapping. Randomized and Maximal absorptance models again give higher efficiencies (maxima of $35.4 \%$ and $36.1 \%$ ) and lower $N_{m p p}(0.62$ and 0.57$)$. In all cases $N_{m p p}$ is higher than in a solar cell which interacts with a full $2 \pi$ hemisphere, indicating photon recycling is more significant here. These calculations corroborate that increasing roughness reduces $N_{m p p}$ yet improves efficiency.

We now consider $\mathrm{CsPbBr}_{3}$ LEDs. We use a reciprocal model to our description of solar cells (see Supplementary Note 5). We present results using the Direct absorptance (here, emittance) model for an LED which emits over a full $2 \pi$ hemisphere unless otherwise stated; we see similar results from other models (Figures S14-S17). We present the number of photon recycling events in the LED, $N$, as a function of thickness, with no charge trapping, in Figure 4a. We consider an applied voltage which gives a luminous emittance of $1000 \mathrm{~lm} \mathrm{~m}^{-2}$ at $100-$ $\mathrm{nm}$ film thickness. Our calculations demonstrate photon recycling occurs significantly more within LEDs than solar cells under operating conditions: $N$ can be as high as 8 here. In analogy to efficiency in a solar cell (cf. Figure 1a), luminous emittance also increases with thickness (Figure S15).

We plot $N$ as a function of applied voltage for different first order trapping rates in a $100-\mathrm{nm}$ emitter film in Figure 4b. Our results demonstrate that for photon recycling to be important (i.e. $N>0.1$ at $10001 \mathrm{~m} \mathrm{~m}^{-2}$ ) charge trapping rates need to be $<10^{6} \mathrm{~s}^{-1}$. Furthermore, Auger recombination reduces $N$ at larger charge densities. 

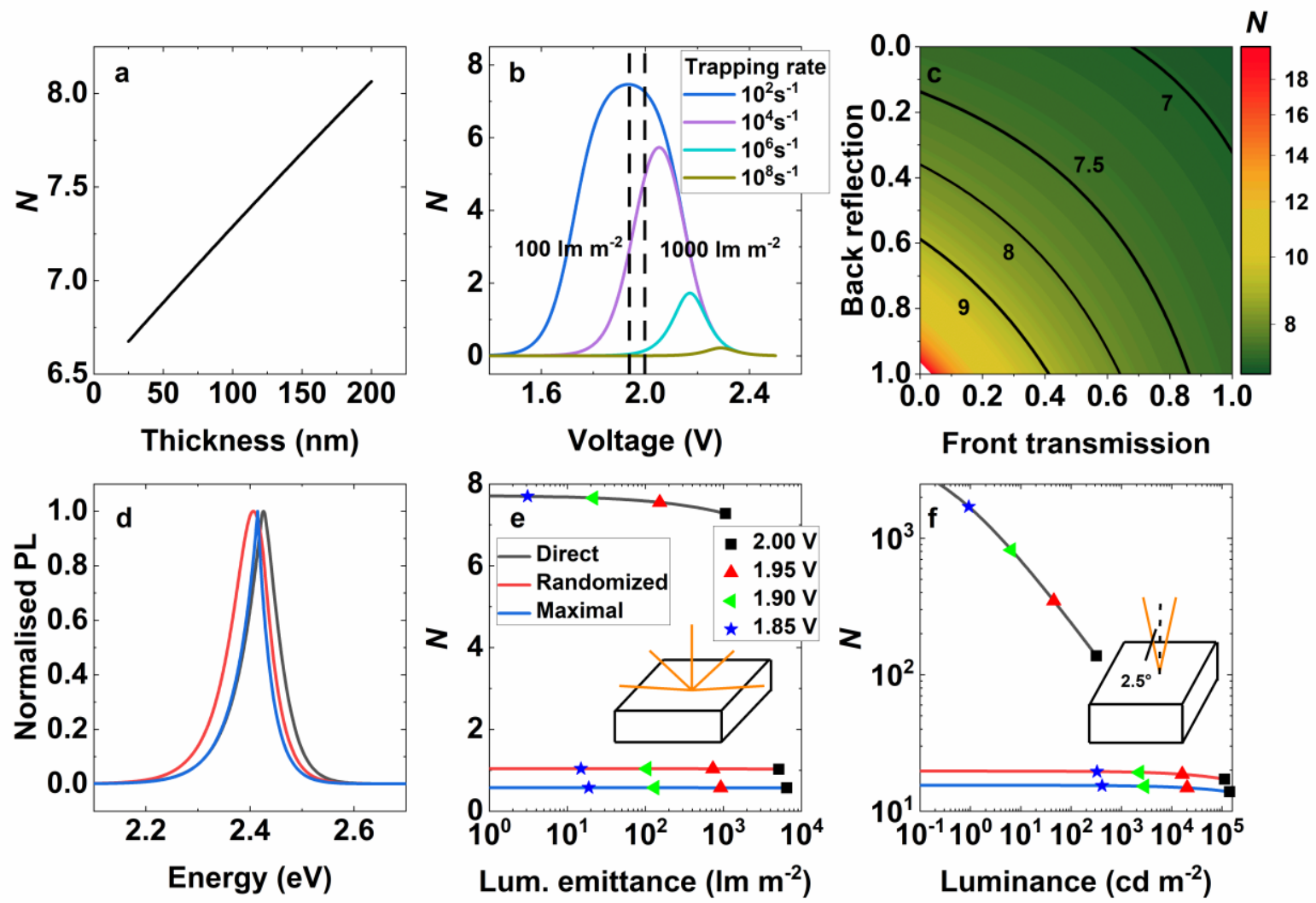

Figure 4. Number of photon recycling events, $N$, in a CsPbBr 3 LED as a function of: a) thickness, for an applied voltage which gives $1000 \mathrm{~lm} \mathrm{~m}^{-2}$ at 100-nm thickness (and no charge trapping), and b) voltage for different charge trapping rates. Voltages corresponding to $100 \mathrm{~lm}$ $\mathrm{m}^{-2}(1.94 \mathrm{~V})$ and $1000 \mathrm{~lm} \mathrm{~m}^{-2}(2.00 \mathrm{~V})$ are marked with dashed lines. $\left.c\right) \mathrm{N}$ as a function of front transmission and back reflection coefficients. d) Normalized photoluminescence spectra for the three emittance models. Legends in e apply to $d$, e and f. e) $N$ and luminous emittance as a function of applied voltage for the three emittance models, with specific voltages marked, for emission into a full $2 \pi$ hemisphere (see schematic). f) The equivalent plot to e) for an LED which emits into a $2.5^{\circ}$ cone about the normal to its surface (see schematic). Calculations in b) to f) are performed on a 100-nm thick film.

We plot $N$ as a function of front transmission and back reflection coefficients in Figure 4c (for a $100-\mathrm{nm}$ thick film with no charge trapping and voltage set to give maximum forward luminous emittance of $1000 \mathrm{~lm} \mathrm{~m}^{-2}$ ). $N$ is equally impacted by the front and rear transmission coefficients as our model is fully symmetric. $N$ varies relatively slowly until front transmission is less than 0.4 and back reflection greater than 0.6. Here $N$ becomes greater than 9 and rapidly increase further for weaker front transmission. We find that forward luminous emittance is 
maximized for maximum front transmission and minimal back transmission (Figure S18), unlike $N$.

We now consider the effects of surface roughness with our three emittance models: Direct, Randomized and Maximal. We note these models give slightly different photoluminescence emission spectra (Figure 4d). We plot $N$ versus luminous emittance (both as a function of voltage) for LEDs which emit into a $2 \pi$ hemisphere in Figure 4e. $N$ is dramatically reduced from greater than 7 for Direct to less than 1 for Maximal emittance. However, Maximal has the highest luminous emittance of $\sim 6000 \mathrm{~lm} \mathrm{~m}^{-2}$, compared to $\sim 1000 \mathrm{~lm} \mathrm{~m}^{-2}$ for the Direct case at the same applied voltage, noting non-radiative Auger recombination reduces luminescence further in the latter case due to increased photon trapping. We find that rough surfaces emit more light and reduce $N$, as was the case for solar cells.

The difference for the three emittance models is even more evident for an LED which only emits $2.5^{\circ}$ about the normal to the LED's surface (Figure $4 \mathrm{f}$, which can be achieved by use of nanostructures [19-22]). For an applied voltage giving a luminance of $100 \mathrm{~cd} \mathrm{~m}^{-2}$ for the Direct model, 44,000 $\mathrm{cd} \mathrm{m}^{-2}$ is achieved with the Maximal emittance model. However, as in the case of solar cells, photon recycling becomes an integral part of all optimized LEDs that only emit into a small solid angle, with $N>10$ in all models. These collective results highlight the reciprocal nature of our model for both light absorption and light emission, and that maximum efficiency (or emitted light) is not necessarily achieved with maximum photon recycling. In conclusion, we present a general framework to quantify the number of photon recycling events, $N$. We apply our model to $\mathrm{MAPbI}_{3}$ solar cells and $\mathrm{CsPbBr} 3 \mathrm{LEDs}$, revealing that even for a highly luminescent (i.e. well-passivated) solar cell $N$ is less than 1 per absorbed solar photon at maximum power point. Conversely, in LEDs $N$ can be as high as 8 at typical LED operating voltages, or even higher for solid angles of emission smaller than $2 \pi$. We show that photon recycling, solar cell efficiency and luminous emittance all increase for thicker cells, cells with reduced charge trapping, and cells with better back reflectors. However, better light management and front transmission for a given thickness increase efficiency/luminosity but decrease $N$. Our results demonstrate that absorptance/emittance should be maximized when optimizing solar cells/LEDs, even if this reduces the number of photon recycling events. 


\section{Acknowledgements}

ARB acknowledges funding from a Winton Studentship, Oppenheimer Studentship and the Engineering and Physical Sciences Research Council (EPSRC) Doctoral Training Centre in Photovoltaics (CDT-PV). MA acknowledges funding from the Marie Skłodowska-Curie actions (grant agreement No. 841386) under the European Union's Horizon 2020 research and innovation programme. SDS acknowledges the Royal Society and Tata Group (UF150033). We thank Luis Pazos-Outón for supplying data for $\mathrm{MAPbI}_{3}$ solar cells. This work was supported by EPSRC grant EP/S030638/1.

\section{Data statement}

The data underlying this manuscript are available at [url to be added in proof].

\section{Conflicts of Interest}

SDS is a Co-Founder of Swift Solar Inc. 


\section{Bibliography}

[1] O. Von Roos, J. Appl. Phys. 54, 1390 (1983).

[2] J. E. Parrott, Sol. Energy Mater. Sol. Cells 30, 221 (1993).

[3] L. M. Pazos-Outon, M. Szumilo, R. Lamboll, J. M. Richter, M. Crespo-Quesada, M. Abdi-Jalebi, H. J. Beeson, M. Vrućinić, M. Alsari, H. J. Snaith, B. Ehrler, R. H. Friend, and F. Deschler, Science (80-. ). 351, 1430 (2016).

[4] C. Cho, B. Zhao, G. D. Tainter, J. Y. Lee, R. H. Friend, D. Di, F. Deschler, and N. C. Greenham, Nat. Commun. 11, 1 (2020).

[5] J. Nelson, The Physics of Solar Cells (Imperial College Press, 2003).

[6] U. Rau, U. W. Paetzold, and T. Kirchartz, Phys. Rev. B 035211, 1 (2014).

[7] T. W. Crothers, R. L. Milot, J. B. Patel, E. S. Parrott, J. Schlipf, P. Müller-Buschbaum, M. B. Johnston, and L. M. Herz, Nano Lett. 17, 5782 (2017).

[8] T. Kirchartz, F. Staub, and U. Rau, ACS Energy Lett. 1, 731 (2016).

[9] R. Brenes, M. Laitz, J. Jean, D. W. Dequilettes, and V. Bulović, Phys. Rev. Appl. 12, (2019).

[10] W. van Roosbbroeck and W. Shockley, Phys. Rev. 94, 1558 (1954).

[11] J. C. De Mello, H. F. Wittmann, and R. H. Friend, Adv. Mater. 9, 230 (1997).

[12] S. D. Stranks, G. E. Eperon, G. Grancini, C. Menelaou, M. J. P. Alcocer, T. Leijtens, L. M. Herz, A. Petrozza, and H. J. Snaith, 342, 341 (2013).

[13] L. M. Pazos-Outón, T. P. Xiao, and E. Yablonovitch, J. Phys. Chem. Lett. 9, 1703 (2018).

[14] D. W. DeQuilettes, S. Koch, S. Burke, R. K. Paranji, A. J. Shropshire, M. E. Ziffer, and D. S. Ginger, ACS Energy Lett. 1, 438 (2016).

[15] M. V. Khenkin, K. M. Anoop, E. A. Katz, and I. Visoly-Fisher, Energy Environ. Sci. 12, 550 (2019).

[16] O. Höhn, T. Kraus, G. Bauhuis, U. T. Schwarz, and B. Bläsi, Opt. Express 22, A715 (2014).

[17] M. Peters, C. Ulbrich, J. C. Goldschmidt, J. Fernandez, G. Siefer, and B. Bläsi, Opt. Express 19, A136 (2011).

[18] C. Ulbrich, M. Peters, B. Bläsi, T. Kirchartz, A. Gerber, and U. Rau, Opt. Express 18, A133 (2010).

[19] G. Lozano, G. Grzela, M. A. Verschuuren, M. Ramezani, and J. G. Rivas, Nanoscale 6, 9223 (2014).

[20] S. Zhang, G. A. Turnbull, and I. D. W. Samuel, Adv. Opt. Mater. 2, 343 (2014).

[21] S. H. Yang, T. J. Huang, and T. H. Liu, J. Lumin. 201, 402 (2018).

[22] J. Dimaria, E. Dimakis, T. D. Moustakas, and R. Paiella, Appl. Phys. Lett. 103, 1 (2013). 